\section{EDUCATION}

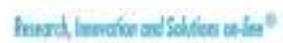

\section{Electronic Journal of Research}

in Educational Psychology

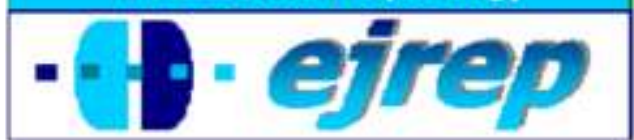

\title{
Consumo autoinformado de alcohol y otras drogas en población universitaria española
}

\author{
Flor Zaldívar Basurto, Francisca López Ríos \\ José Manuel García Montes, Antonio Molina Moreno
}

Departamento de Personalidad, Evaluación y Tratamiento Psicológico,

Universidad de Almería

\section{España}

Correspondencia: Francisca López Ríos. Facultad de Psicología. Universidad de Almería. Carretera de Sacramento s/n. 04120 Almería. España. E-mail: flrios@ual.es

(C) Education \& Psychology I+D+i and Editorial EOS (Spain) 


\section{Resumen}

Introducción. Los objetivos de este estudio son explorar el consumo de alcohol y otras drogas en universitarios/as y comprobar si existen diferencias en consumo según el sexo.

Método. Se trata de un estudio descriptivo mediante encuesta. Se ha evaluado el consumo de drogas en 506 alumnos/as de la Universidad de Almería (España). Dicha evaluación se ha llevado a cabo a través de un autoinforme elaborado expresamente para esta investigación, con el que se ha recabado información acerca de la intensidad y frecuencia de consumo de alcohol y otras drogas. Otros instrumentos de medida del consumo de drogas utilizados son el $C A G E$ (alcohol), el $A A S$, el $A P S$, el $A U D I T$ y el $M A C$.

Resultados. Los resultados muestran que el alcohol es la sustancia preferida, más consumida y con la que se alcanzan con más frecuencia estados de embriaguez (en torno al 70\% de los sujetos). La segunda sustancia más consumida, preferida y con la que se han "colocado" más frecuentemente es el cannabis. Además, en el último mes, el 86,2\% de los sujetos ha consumido alcohol, el 32, 6\% cannabis y el 3,0\% drogas de diseño. En relación con el género, los varones han consumido más alcohol y más cannabis que las mujeres en la última semana.

Discusión. Estos resultados coinciden con los obtenidos en otras universidades españolas, pero superan los índices de prevalencia reseñados por otros estudios con respecto a la población joven general y con respecto a la población estudiante entre 14 y18 años.

Palabras clave: universitarios, drogas, alcohol, estudio descriptivo mediante encuesta. 


\title{
Self-reported consumption of alcohol and other drugs in the university population
}

\begin{abstract}
Introduction. The aims of this study are to explore the consumption of alcohol and other drugs in university students and to check if gender differences exist in the consumption of these substances
\end{abstract}

Method. Descriptive study through self-reports. Drugs consumptions has been evaluated in 506 students from the University of Almería (60, 9\% women and 34, 6\% men). Such evaluation has been carried out through a self-report measure elaborated specifically for this research gathering information about intensity and frequency of alcohol and other drugs. Others measure instruments used has been the $C A G E$ (alcohol), the AAS, the APS, the AUDIT and the $M A C$.

Results. Some of the results collected show that alcohol is the preferred substance, the more consumed and the one with which university students achieve more frequently intoxication states (around $70 \%$ of subjects). The second substance more consumed and preferred by students and with which students "get high" is cannabis. Also if the last month of consumption is analyzed, 82, 6\% of subjects have consumed alcohol, 32, 6\% cannabis and 3, 0\% design drugs. In relation to gender, men have consumed significantly more alcohol and cannabis than women if the last week is analyzed.

Discussion and Conclusion: These results are similar to others obtained in different Spanish universities, but exceed the prevalence rates indicated in other studies regarding general young population and with the students among 14 and 18 years.

Keywords: universitary population, drugs, alcohol, descriptive study through self- 


\section{Introducción}

Existe una preocupación creciente por el consumo de drogas en nuestro país, muy especialmente por el consumo por parte de los jóvenes, ya que se entiende que las consecuencias del consumo sobre el desarrollo biológico, psicológico y social en edades tempranas pueden ser especialmente dramáticas e irreversibles, (Caspari, 1999; Iversen, 1999; Lizasosain, Moro y Lorenzo, 2001; Saez García-Portilla, Martínez y cols, 2003; Sainz, García-Portilla, Martínez y cols., 2003). Puede que parte esta preocupación venga justificada además porque en las últimas décadas hemos asistido a una ampliación del tipo y cantidad de sustancias disponibles en el mercado ilegal de drogas al tiempo que se han consolidado patrones de ocio caracterizados por concentraciones más o menos numerosas de jóvenes los fines de semana al aire libre en las que junto al alcohol se comparte y extiende el consumo de otras drogas como el cannabis, el éxtasis MDMA, los alucinógenos, el MDA, la cocaína, etcétera (Calafat, Fernández, Juan y Becoña, 2005; Infante, Barrio y Martin, 2003; Rodríguez, Agulló y Agulló, 2003).

Desde hace años contamos en España con estudios sistemáticos, realizados a través del Observatorio Español de Drogodependencias y de los diversos planes autonómicos sobre drogas, que proporcionan información regular acerca del consumo de sustancias adictivas por parte de la población general, por sectores de edad y diferenciada por sexos. También se han desarrollado estudios sistemáticos sobre el consumo del estudiantes entre 14 y 18 años, de enseñanza secundaria, bachillerato y ciclos formativos medios, (ESTUDES, 1994-2008). De este modo, y brevemente, siguiendo la EDADES (2008), el Informe del Observatorio Español de Drogas (OED, 2007) y la ESTUDES (2008) resumimos los datos más actuales, así como la tendencia epidemiológica.

El alcohol es la principal sustancia de consumo en nuestro país. Tanto es así que, en 2008, el 76,1\% de los varones españoles y el 51,4\% de las mujeres habían consumido alcohol en los últimos 30 días. El mayor consumo semanal se situaba entre los 25 y los 29 años y la edad media de inicio se encontraba en los 16,9 años. El consumo diario de alcohol estaba en el 15,3\% de la población, fundamentalmente entre los 30 y 65 años. Cabe destacar que entre los 20 y 24 años el 5,8\% de la población se consideraba bebedor de riesgo o de gran riesgo y entre 30 y 34 años, el 6,2\%. Sin embargo, se ha ido observando una tendencia hacia la estabilización en el consumo en 2005 (EDADES, 2006) hasta llegar en 2008 a un descenso de 
aproximadamente 3 puntos en la prevalencia de consumo por edades y sexos (EDADES, 2008). En estudiantes entre 14 y 18, el consumo de alcohol en los últimos 12 meses es del $72,9 \%$, en los últimos 30 días es del 58,5\% y la edad de inicio se sitúa en los 13,7 años (ESTUDES, 2008).

Por su parte, el cannabis es la sustancia ilegal de uso más extendido. De hecho, en el año 2001 un 24\% de los españoles entre 15 y 64 años lo había probado alguna vez en su vida, un 6,5\% lo había consumido en los últimos 30 días y un 1,6\% lo consumía a diario (OED) (2003). La tendencia en las prevalencias entre 1995 y 2001 muestra un incremento del consumo en los distintos periodos (por ejemplo, desde un 3,1\% a un 6,5\% en los últimos 30 días). El consumo mensual y diario ha seguido aumentando en 2003 y 2005 (por ejemplo, el consumo diario ha pasado al 2,0\%) aunque se han estabilizado los consumos experimentales (EDAD, 2006). La edad de inicio en el consumo de cannabis se situaba en 2001 en 18,4 años, manteniéndose en 18,3 años en 2005 (EDAD, 2006) y de 18,5 en el año 2008. En el último estudio se ha invertido la tendecia de la prevalecia observándose una reducción de aproximadamente dos puntos tanto por edades como por sexos. Entre 14 y18 años, el consumo de cannabis en los últimos 12 meses es de 30,5\%, en los últimos 30 días es del 20,1\% y la edad de media de inicio se sitúa en los 14,6 años (ESTUDES, 2008).

En lo que respecta al éxtasis o MDMA se considera la droga recreativa más consumida en España. Tanto es así que en el año 2001 un 6,2\% de los varones y un 2,2\% de las mujeres de entre 15 y 64 años habían probado el éxtasis alguna vez en su vida mientras que en los últimos 30 días lo habían hecho el 1,2\% de los varones y el 0,2\% de las mujeres. Los grupos de edad en los que el consumo es mayor en los últimos 30 días son los situados entre 15 y 19 años $(2,8 \%$ en varones y $0,6 \%$ en mujeres) y entre 20 y 24 años (2,6\% en varones y $0,9 \%$ en mujeres) (OED) (2003) con consumo creciente en el en el periodo 1995 - 2001. En 2008 se observa un descenso en el consumo tanto en la prevalencia anual como en el consumo en los últimos 30 días en varones con respecto a los datos de 2005 (EDADES, 2008). La edad media de inicio se mantiene en torno a los 20 años (EDADES, 2005, 2008). El éxtasis es consumido por un $1,9 \%$ de los estudiantes de secundaria en los últimos 12 meses y por un 1,1 en los últimos 30 días. La edad de inicio se sitúa en 15,2 años.

En el caso de la cocaína (en polvo), en el año 2001, un 2,6\% de los españoles entre 15 y 64 años había consumido cocaína en los últimos 12 meses y un 1,4\% lo había hecho en los 
últimos 30 días. En este caso se observa un importante aumento del consumo entre 1999 y 2001 (OED) (2003). En el año 2005 el consumo de cocaína se había situado en un 3\% de los españoles y un 1,6\% en el último mes. En 2008 estas cifras se han mantenido. Existen importantes diferencias entre sexos. Por ejemplo, en 2008 el consumo en los últimos 30 días en varones se situó en el 2,9\% frente al 0,7\% de mujeres. La edad de inicio promedio permanece estable en 20,6 años y el grupo de edad en que dicho consumo es más frecuentes es entre 15 y 34 años (EDAD, 2006). Entre los 14 y 18 años, las cifras de consumo de cocaína son del 3,6\% y del $2 \%$ respectivamente, según se trate de los últimos 12 meses o de los últimos 30 días. La edad media de inicio en este grupo de edad está en los 15, 3 años.

Sustancias menos consumidas son las anfetaminas o speed, (0,5\% en varones y $0,1 \%$ en mujeres en los últimos 30 días en 2008), la cocaína base (en la que se observa un incremento del consumo en el último informe EDADES) o la heroína (que se mantiene en un consumo entre el 0 y $0,1 \%$ ). En el caso de estudiantes entre 14 y 18 años la heroína es consumida por un $0,7 \%$ y por un $0,6 \%$, respectivamente, y la edad en el inicio de su consumo es de 14,3 años. Los alucinógenos y las anfetaminas están en torno al 2,5\% en los últimos 12 meses y en un 1,2\% en el último año, con 15,4 años como edad de inicio.

En resumen, los datos mencionados señalan estabilización de las prevalencias de las diferentes sustancias, incluso una inversión en la tendencia. Además se mantiene la edad promedio de inicio del consumo. Si bien es cierto que cuando se acota la muestra a población estudiante entre 14 y 18 años, los índices de prevalencia son mayores que cuando se considera la población general. Este hecho señala claramente que el inicio y estabilización del consumo de drogas se produce en edades escolares y que los centros educativos son contextos apropiados para desarrollar programas preventivos.

De hecho, otros informes europeos permiten establecer diferencias en el consumo juvenil de alcohol y otras drogas en función de las sustancias disponibles en cada país. Así, según el European Monitoring Centre for Drugs and Drug Addiction (2009), en población de 16 años, el consumo principal en toda Europa es el alcohol con cifras similares a las españolas, o superiores. Sin embargo la segunda droga ilegal en consumo varía entre el cannabis y los inhalables. Es decir, cambian las sustancias, pero los índices de consumo se mantienen muy similares. 
Sin embargo, no contamos con estudios sistemáticos del consumo de drogas en población universitaria, que nos permita valorar -al menos en parte-, la evolución del consumo en el siguiente eslabón educativo, es decir, si durante el periodo universitario se producen nuevas incorporaciones al consumo de sustancias adictivas. En este contexto, existen algunos trabajos de epidemiología de consumo en población joven en el ámbito universitario. Así, en 1987 se publicó un estudio sobre el consumo de alcohol en la Universidad de Valladolid (Queipo, Álvarez y Velasco, 1986) en el que se informaba de que el 88,9\% de los varones estudiantes y el 73,3\% de las mujeres consumía alcohol los fines de semana, sin que se mencionaran otras sustancias. Laporte, (1979) encontraron en la Universidad de Barcelona, un consumo de alcohol en los estudiantes menor que en la población general, un $22 \%$ de sujetos que habían consumido anfetaminas en los últimos 6 meses y un 9,6\% había consumido cannabis. Más recientemente, Viña y Herrero (2004) llevaron a cabo un estudio con alumnos de la Universidad de la Laguna, e informaron que el $86,3 \%$ de los varones y el $73,1 \%$ de las mujeres consumían alcohol alguna vez al mes, y el 30,2\% de los hombres y el 13, 2\% de las mujeres consumieron cannabis en el mismo periodo. En el caso de la cocaína, había consumido en el último mes el $3,5 \%$ de los varones y el 2,2\% de las mujeres.

En relación con el alcohol, encontramos cifras similares en estudiantes de medicina de la Universidad de Irlanda (Boland, Fitzpatrick, Scallan, et al., 2006), aunque con un menores diferencias entre varones y mujeres y una marcada tendencia al incremento del consumo de alcohol en los últimos 30 años. Resultados similares han sido hallados en un estudio llevado a cabo en el Reino Unido con estudiantes de segundo curso de diferentes titulaciones en 10 de sus universidades (Webb, Ashton, Kelly y Kamali, 1996). Como dato a destacar está el consumo regular (semanal) de cannabis por el 19.8\% de los sujetos encuestados y el de anfetaminas por un $3,5 \%$. Otros informes europeos permiten establecer diferencias en el consumo juvenil de alcohol y otras drogas en función de las sustancias disponibles en cada país. Así, según el European Monitoring Centre for Drugs and Drug Addiction (2009), en población de 16 años, el consumo principal es el alcohol con cifras similares a las españolas, o superiores. Sin embargo la segunda droga ilegal en consumo varía entre el cannabis y los inhalables. No obstante, no contamos con los índices de consumo en contextos universitarios.

En resumen, los estudios señalados apuntan a un mayor consumo de alcohol, cannabis y cocaína por parte de jóvenes universitarios que en la población general. Sin embargo, para poder extraer conclusiones acerca de los patrones de consumo en población universitaria ne- 
cesitaríamos estudios sistemáticos que aportaran dicha información y que garantizaran la validez del autoinforme de los sujetos evaluados.

\section{Objetivos}

En este sentido, el estudio que se describe tiene dos objetivos. Por un lado, comprobar cuáles son los índices de consumo de los jóvenes universitarios de diferentes sustancias (alcohol, cannabis, drogas de diseño, alucinógenos, cocaína, anfetaminas y heroína), y por otro, comprobar si existen diferencias en los parámetros de consumo entre varones y mujeres, además de comprobar si hay correspondencia entre los resultados obtenidos en el autoinforme generado para este investigación y los obtenidos a través de otras escalas validadas de consumo.

\section{Método}

\section{Participantes}

La muestra de sujetos participantes en este estudio son 506 estudiantes, de los cuales 308 son mujeres $(60,9 \%)$ y 198 varones $(34,6 \%)$, todos ellos estudiantes de la Universidad de Almería y procedentes de 15 titulaciones diferentes. La edad media de los sujetos es de 20,9 años, con una desviación típica de 2,4 años y con una mediana de 21 años. El rango de edades se sitúa entre 17 y 35 años.

\section{Diseño y análisis de datos}

Se trata de un estudio de población mediante encuesta de población probabilística de carácter transversal (Montero y León, 2005), con comparaciones en función del sexo. Los análisis estadísticos utilizados son análisis descriptivos de distribución de porcentajes y comparaciones través del estadístico Chi-cuadrado.

\section{Variables e instrumentos}

En este estudio se ha utilizado como instrumento de evaluación un autoinforme o cuestionario autoadministrado sobre consumo de drogas que consta de dos tipos de escalas: 
un Autoinforme de consumo de alcohol y otras drogas, (elaborada expresamente para este estudio) y escalas validadas de consumo.

1) Autoinforme de Consumo de Alcohol y Otras Drogas (diseñado para esta investigación). Es un autoinforme compuesto por preguntas directas sobre consumo de alcohol y otras sustancias. Esta escala de consumo evalúa: a) el consumo de drogas durante el fin de semana; b) sustancias que han sido probadas (alguna vez, durante el último año, último mes, último fin de semana, última vez que "te colocaste"); c) sustancias (o combinación de éstas) que se consumen con mayor frecuencia; d) sustancias favoritas; y e) frecuencia de consumo de las distintas sustancias. Los resultados del informe eran corroborados a través de análisis de orina.

2) AUDIT (Development of the Alcohol Use Disorders Identification Test, Saunders, Aasland, Babor, De la Fuente y Grant, 1993). Versión española de Rubio, Bermejo, Caballero y Santo Domingo (1998). Este test, elaborado por la Organización Mundial de la Salud, detecta bebedores de riesgo. Consta de 10 ítems que exploran el consumo de alcohol del sujeto así como los problemas derivados del mismo. La puntuación se obtiene sumando los 10 ítems y las categorías establecidas son: entre 8 y 15 puntos, nivel medio de problemas con el alcohol; puntuaciones mayor de 15 indicaría nivel de riesgo de desarrollar problemas con el alcohol (Babor, Higgins-Biddle, Saunders y Monteiro, 2001).

3) AAS (Addiction Acknowlegement Scale -MMPI-2- Weed, Butcher, McKenna y BenPorath, 1992; version española de Hathaway \& McKinley, 1999). Es una escala de reconocimiento de la adicción de alcohol y otras drogas, consta de 13 ítems dicotómicos (verdadero/falso) de modo que a mayor puntuación mayor abuso de sustancias. Una puntuación directa de 4 , tanto en varones como en mujeres, es significativa de adicción.

Es una escala de reconocimiento de adicción.

4) Escala CAGE de alcohol (Cage Alcohol Intervies Scale, Mayfield, McLeod y Hall, 1974) Versión española de Rodríguez- Martos, Navarro, Vecino y Pérez, 1986). Es un instrumento para la detección de alcoholismo cuyas preguntas deben intercalarse en un autoinforme más amplio. Consta de 4 preguntas. Una puntuación de 1 se considera indicativa de problemas con el alcohol, y puntuaciones de 2-4 indican dependencia alcohólica. En este trabajo se ha utilizado tambien una adaptación del CAGE para evaluar el consumo de cannabis y cocaína, mo- 
dificando los ítems que evalúan consumo de alcohol para que evalúen estas sustancias $(\mathrm{Mu}-$ ñoz, Roa, Pérez, Santos-Olmo, \& de Vicente, 2002).

5) APS (Addiction Potential Scale-MMPI-2-, Weed, Butcher, McKenna y Ben-Porath, 1992). Esta escala consta de 39 ítems de verdadero o falso incluidos en el MMPI-2, destinados a detectar sujetos que, potencialmente, desarrollarán problemas de adicción. Es una escala indirecta, es decir, sus ítems están camuflados pues no preguntan directamente sobre consumo de sustancias, sino que se relacionan con dimensiones de la personalidad y situaciones vitales asociadas con el abuso de sustancias. Las puntuaciones elevadas sugieren alta probabilidad de abuso de sustancias y, aunque no hay un punto de corte definido, una puntuación directa mayor de 28 en varones y 27 en mujeres indicaría problemas con el uso de sustancias.

6) MAC (McAndrew Alcoholism Scale - MMPI-2, McAndrew, 1965). Es una escala camuflada que consta de 46 ítems de formato de respuesta de verdadero/falso, referidos a características generales del estilo de vida y patrones de personalidad. Es un indicador de alcoholismo potencial. Un punto de corte de 24 para alcoholismo discrimina adecuadamente al $81.5 \%$ de la población.

\section{Procedimiento}

La captación de participantes se realizó a partir del anuncio del estudio a través de carteles repartidos por el Campus de la Universidad de Almería. Los alumnos y alumnas interesados acudían al punto de información en el que se les informaba sobre las condiciones de su participación en el mismo: debían acudir un día y una hora (concretamente en lunes para detectar consumo en el fin de semana previo a la cita), completar un cuestionario sobre personalidad, salud y hábitos de vida en el que se incluía el consumo de alcohol y otras drogas, tras cumplimentar el cuestionario debían proporcionar una muestra de orina. Como contraprestación recibirían la cantidad de $10 €$. Tras mostrar su acuerdo se les emplazaba al día, hora (9h.) y lugar en que se completaba el cuestionario, la recogida de muestras de orina y el pago pactado. 


\section{Resultados}

Los resultados de consumo obtenidos mediante la Escala de Consumo de Alcohol y Otras Drogas tomando como referencia distintos periodos previos al momento de la evaluación, se muestran en la tabla 1.

Tabla 1. Porcentaje de sujetos que han consumido diferentes sustancias en distintos periodos.

\begin{tabular}{|c|c|c|c|c|c|c|c|c|c|c|c|c|}
\hline & \multicolumn{3}{|c|}{ Último año } & \multicolumn{3}{|c|}{ Último mes } & \multicolumn{3}{|c|}{ Última semana } & \multicolumn{3}{|c|}{ Último fin de semana } \\
\hline Sustancia & $\begin{array}{c}\text { Mujeres } \\
(\%)\end{array}$ & $\begin{array}{c}\text { Hombres } \\
(\%)\end{array}$ & Total (\%) & $\begin{array}{c}\text { Mujeres } \\
(\%)\end{array}$ & $\begin{array}{c}\text { Hombres } \\
(\%)\end{array}$ & Total (\%) & $\begin{array}{c}\text { Mujeres } \\
(\%)\end{array}$ & $\begin{array}{c}\text { Hombres } \\
(\%)\end{array}$ & Total (\%) & $\begin{array}{c}\text { Mujeres } \\
(\%)\end{array}$ & $\begin{array}{c}\text { Hombres } \\
(\%)\end{array}$ & Total $(\%)$ \\
\hline Alcohol & 91.2 & 94.9 & 92.7 & 85.1 & 87.9 & 86.2 & 67.2 & 77.3 & 71.1 & 54.2 & 65.7 & 58.7 \\
\hline Porros & 46.8 & 56.6 & 50.6 & 29.5 & 37.4 & 32.6 & 20.8 & 33.3 & 25.7 & 17.9 & 28.8 & 22.1 \\
\hline D. de diseño & 7.5 & 5.6 & 6.7 & 3,6 & 2.0 & 3.0 & 1.3 & 1.0 & 1.2 & 1.3 & - & 0.8 \\
\hline Anfetaminas & 4.2 & 4.5 & 4.3 & 0.6 & 0.5 & 0.6 & 0.3 & - & 0.2 & - & - & - \\
\hline Cocaína & 11.0 & 13.1 & 11.9 & 3.6 & 3.5 & 3.6 & 1.3 & 2.5 & 1.8 & 1.3 & 1.5 & 1.4 \\
\hline Alucinógenos & 3.2 & 2.5 & 3.0 & 0 & 0 & 0 & 0.3 & 0 & 0.2 & - & - & - \\
\hline Heroína & 0.3 & 1.5 & 0.8 & 0 & 1.0 & 0.4 & 0 & 0.5 & 0.2 & 0 & 0.5 & 0.2 \\
\hline
\end{tabular}

Así, el último año, el 92,7\% de los sujetos ha consumido alcohol, el 50,6\% ha fumado porros (cannabis), el 6,7\% ha probado las drogas de diseño, el 4,3\% ha consumido anfetaminas, el 11,9\% ha consumido cocaína, el 3\% ha probado los alucinógenos y el 0,8\% ha tenido contacto, al menos una vez, con la heroína. En el último mes, el 86,2\% de los sujetos han tomado alcohol, el 32,6\% ha fumado cannabis, el 3\% ha consumido drogas de diseño, el 0,6\% ha consumido anfetaminas, el 3,6\% ha consumido cocaína, no se han consumido alucinógenos y un 0,4\% ha tenido contacto con la heroína. La prevalencia referida al consumo en la semana previa a la evaluación, los resultados señalan que el 71,1\% de los sujetos ha consumido alcohol y el $25 \%$ ha consumido cannabis, cocaína el 1,8\% y drogas de diseños el 1,2\%. En este caso aparecen diferencias estadísticamente significativas entre sexos en el consumo de alcohol (Chi-cuadrado, 5,947; p=0,015) y en el consumo de porros (Chi-cuadrado, 
9,950; $\mathrm{p}=0,002)$, de modo que los varones consumen significativamente más alcohol y porros que las mujeres. En el fin de semana previo a la evaluación del consumo de drogas, el alcohol sigue siendo la sustancia más consumida con un 58,7\% de los sujetos, seguido por el consumo de porros por un 22,1\% de los sujetos. La tercera sustancia más consumida es la cocaína con un porcentaje del 1,4. En este caso también se aprecian diferencias estadísticamente significativas entre sexos tanto en alcohol (Chi-cuadrado, 6,501; $\mathrm{p}=0,011)$ como en porros (Chi-cuadrado, 8,355; $\mathrm{p}=0.004$ ), siendo significativamente mayor el consumo de ambas sustancias en varones que en mujeres.

En relación con los resultados relativos a la intensidad del consumo de las diferentes sustancias evaluadas, entendida como la frecuencia con la que se alcanzan estados de embriaguez o "estar colocado" con cada una de ellas, los datos señalan que el alcohol es la sustancia con que los sujetos se "colocan" con más frecuencia, seguido por el alcohol sumado a otras drogas y por el consumo de cannabis. Menos frecuente es alcanzar estados de embriaguez a través del consumo aislado de cocaína, pastillas (drogas de diseño), o heroína (ver tabla 2).

Tabla 2. Porcentaje de sujetos que han consumido las distintas sustancias hasta "colocarse". Establecimiento de diferencias en función del sexo.

\begin{tabular}{|c|c|c|c|c|c|c|c|c|c|c|c|c|c|c|c|c|c|c|}
\hline & \multicolumn{3}{|c|}{ Alcohol } & \multicolumn{3}{|c|}{ Alcohol +porros } & \multicolumn{3}{|c|}{ Porros } & \multicolumn{3}{|c|}{ Cocaína } & \multicolumn{3}{|c|}{ Pastillas } & \multicolumn{3}{|c|}{ Heroína } \\
\hline & $\begin{array}{l}M u j . \\
(\%)\end{array}$ & $\begin{array}{l}\text { Homb } \\
\text { (\%) }\end{array}$ & $\begin{array}{l}\text { Total } \\
(\%)\end{array}$ & $\begin{array}{l}M u j . \\
(\%)\end{array}$ & $\begin{array}{l}\text { Homb } \\
(\%)\end{array}$ & $\begin{array}{l}\text { Total } \\
(\%)\end{array}$ & $\begin{array}{l}M u j . \\
(\%)\end{array}$ & $\begin{array}{l}\text { Homb } \\
(\%)\end{array}$ & $\begin{array}{l}\text { Total } \\
(\%)\end{array}$ & $\begin{array}{l}\text { Muj. } \\
(\%)\end{array}$ & $\begin{array}{l}\text { Homb } \\
(\%)\end{array}$ & $\begin{array}{l}\text { Total } \\
(\%)\end{array}$ & $\begin{array}{l}M u j . \\
(\%)\end{array}$ & $\begin{array}{l}\text { Homb } \\
\text { (\%) }\end{array}$ & $\begin{array}{l}\text { Total } \\
(\%)\end{array}$ & $\begin{array}{l}M u j . \\
(\%)\end{array}$ & $\begin{array}{l}\text { Homb } \\
(\%)\end{array}$ & $\begin{array}{l}\text { Total } \\
(\%)\end{array}$ \\
\hline Nunca & 46.1 & 28.8 & 39.3 & 62,7 & 48.5 & 57.1 & 72.7 & 65.7 & 70.0 & 94.5 & 94.4 & 94.5 & 43.2 & 36.1 & 40.7 & 99.4 & 99.0 & 99.2 \\
\hline Menos de 1 vez al mes & 14.0 & 13.6 & 13.8 & 12.3 & 12.1 & 12.3 & 7.5 & 6.1 & 6.9 & 3.2 & 2.0 & 2.8 & 15.3 & 14.4 & 15.0 & 0.6 & 0.5 & 0.6 \\
\hline Mensualmente & 12.0 & 15.7 & 13.4 & 8.4 & 11.6 & 9.7 & 4.9 & 4.0 & 4.5 & 1.0 & 3.0 & 1.8 & 13.1 & 11.3 & 12.5 & & 0.5 & 0.2 \\
\hline Semanalmente & 26.0 & 41.9 & 32.2 & 14.6 & 25.8 & 19.0 & 6.8 & 12.1 & 8.9 & 0.6 & 0.5 & 0.6 & 26.2 & 38.1 & 34.4 & & & \\
\hline A diario & 1.9 & $-\cdots---$ & 1.2 & 1.9 & 2.0 & 2.0 & 8.1 & 12.1 & 9.7 & 0.6 & --- & 0.4 & 2.2 & ---- & 1.4 & & & \\
\hline $\begin{array}{l}\text { Chi cuadrado } \\
\text { Sig. }\end{array}$ & \multicolumn{3}{|c|}{$\begin{array}{c}23.758 \\
.000\end{array}$} & \multicolumn{3}{|c|}{$\begin{array}{c}13.397 \\
.009\end{array}$} & & \multicolumn{3}{|l|}{---} & \multicolumn{3}{|c|}{-----} & \multicolumn{3}{|c|}{--------------- } \\
\hline
\end{tabular}


Tomando como referencia las sustancias consumidas por los sujetos la última vez que alcanzaron estados de embriaguez, en la tabla 3 se muestra que en torno al $70 \%$ de la muestra consumió alcohol, seguido por cannabis (porros) y por ambas sustancias conjuntamente. No se aprecian diferencias estadísticamente significativas entre sexos.

Tabla 3. Frecuencia y porcentaje de sujetos que han consumido diferentes sustancias la última vez que se "colocaron" en función del sexo.

\begin{tabular}{|l|l|l|l|l|}
\hline & $\begin{array}{l}\text { mujeres } \\
\mathrm{n}=308\end{array}$ & $\begin{array}{l}\text { hombres } \\
\mathrm{n}=198\end{array}$ & $\begin{array}{l}\text { total } \\
\mathrm{n}=506\end{array}$ & \multirow{2}{*}{ Sig. } \\
\hline Ninguna & $21.8(67)$ & $12.6(25)$ & $18.2(92)$ & \\
\hline Alcohol & $66.6(205)$ & $75.3(149)$ & $70.0(354)$ \\
\hline Porros & $7.8(24)$ & $6.6(13)$ & $7.3(37)$ & \\
\hline Drogas de diseño & $1.0(3)$ & - & $.6(3)$ & \multirow{2}{*}{-----} \\
\hline Anfetaminas & - & - & - & \\
\hline Cocaína & $.3(1)$ & $.5(1)$ & $.4(2)$ & \\
\hline Alucinógenos & - & - & - & \\
\hline
\end{tabular}

Los resultados obtenidos en las escalas validadas utilizadas en este estudio se muestran en la tabla 4. Teniendo en cuenta los baremos de cada una de ellas se pueden destacar los siguientes aspectos. En primer lugar, la mediana obtenida con el cuestionario CAGE (alcohol) ha sido 1 , valor que indicaría que el $50 \%$ de los participantes podrían manifestar problemas con el alcohol. Más aún, el valor obtenido en el percentil 75 ha sido 2, lo que significa que el $25 \%$ de la muestra podría presentar indicios de dependencia alcohólica. Estos resultados no se producen en el CAGE para el caso cocaína y cannabis, donde no se encuentran indicios de problemas por el uso de dichas sustancias en tal porcentaje de la muestra.

Tabla 4. Mediana y percentiles en los cuestionarios: CAGE, (alcohol, cannabis y cocaína), AUDIT, APS, MAC, ASS y Cantidad de Consumo.

\begin{tabular}{|l|r|r|r|r|r|r|r|r|}
\hline & $\begin{array}{l}\text { CAGE } \\
\text { Alcohol }\end{array}$ & $\begin{array}{l}\text { CAGE } \\
\text { Cannabis }\end{array}$ & $\begin{array}{l}\text { CAGE } \\
\text { Cocaina }\end{array}$ & Audit & APS & MAC & ASS & $\begin{array}{l}\text { CANTIDAD } \\
\text { CONSUMO }\end{array}$ \\
\hline Percentil (25) & 0 & 0 & 0 & 2 & 20 & 16 & 1 & 4 \\
\hline Mediana & 1 & 0 & 0 & 5 & 23 & 19 & 2 & 8 \\
\hline Percentil (75) & 2 & 0 & 0 & 8 & 25 & 22 & 3 & 14 \\
\hline
\end{tabular}


En segundo lugar, los puntos de corte en la escala AUDIT indicarían un perfíl de bebedores de riesgo que están situados, en el caso de los hombres, en el rango de 8-12 y de 6-12 en el caso de las mujeres. La puntuación obtenida en el percentil 75 ha sido de 8 lo que indicaría que el $25 \%$ de la muestra encuestada presentaría un perfil que se calificaría como bebedores de riesgo, resultado que coincide con el obtenido en el CAGE (alcohol).

En tercer lugar, en la escala de potencial de adicción (APS) una puntuación en torno a 28 para varones y 27 para mujeres, indicaría dificultades con el uso de sustancias (refiriéndose a alcohol y a otras drogas). El valor obtenido en el percientil 75 es de 25 lo que indicaría que el $25 \%$ de los sujetos obtienen puntuaciones cercanas al diagnóstico de problemas con ciertas sustancias. En cuarto lugar, el percentil 75 en la en la escala MAC es de 22, valor muy próximo al punto de corte de dicha escala que es de 24 que discriminaría adecuadamente a sujetos consumidores de alcohol en un porcentaje del $81,5 \%$. Finalmente, en la escala de reconocimiento de la adicción (AAS) se interpreta la puntuación máxima de 4 como indicativa de abuso de sustancias. El percentil 75 tiene un valor de 3 muy cercano al diagnóstico de abuso de sustancias derivado por la escala.

\section{Discusión}

Los resultados sobre prevalencia en el consumo de sustancias adictivas obtenidos en este estudio confirman el predominio del alcohol como sustancia más consumida en todos los parámetros considerados: último año, último mes, última semana, último fin de semana y la última vez que los sujetos alcanzaron niveles de embriaguez o se "colocaron". Tanto es así que los resultados obtenidos muestran que el 71,1\% de los sujetos han consumido alcohol en la última semana, lo que da cuenta de que tal consumo es habitual. Tal vez, el resultado más llamativo es que el $41,9 \%$ de los varones y el 26,0\% de las mujeres consumen alcohol hasta alcanzar la embriaguez cada semana. Por otro lado, comparando estos resultados de este estudio con los obtenidos por el OED (2003) y por la EDADES (2008), podemos señalar que la proporción de jóvenes universitarios que consumen alcohol con una frecuencia semanal es muy superior al consumo de la población general. Además, la prevalencia de consumo de alcohol en los últimos 30 días es superior en los sujetos evaluados en este estudio que en los jóvenes entre 20-24 años y entre 25-29 años (OED, 2003; EDADES, 2008) y muy superior al consumo por parte de los estudiantes entre 14-18 años (ESTUDES, 2008). 
Los resultados relativos al consumo de cannabis, señalan un consumo mucho más extendido en la población universitaria almeriense que en la población española general, en las distintos periodos de consumo evaluados. Por otro lado, el consumo de cannabis en las distintas prevalencias es superior en la población universitaria evaluada que en el conjunto de la población joven (entre 20 y 29 años) (OED,2003; EDADES, 2008). Si comparamos estos resultados con los derivados del ESTUDES (2008), podemos comprobar que el consumo de cannabis en universitarios en los últimos 30 días es superior en un 10\% al de los jóvenes estudiantes entre 14 y 18 años.

En el caso de la cocaína, los resultados de este estudio muestran que su consumo es fundamentalmente ocasional, es decir, es una sustancia que pasa de una prevalencia anual del $11,9 \%$ a una prevalencia semanal del $1,8 \%$, Con todo, el consumo en universitarios es superior al de la población joven en general y al de la población estudiante entre 14 y 18 años $(3,6 \%$ con respecto al $2 \%)$.

En relación con las drogas de diseño, la extensión de su consumo pasa de un 6,7\% de la población en los últimos 12 meses a un 1,2 en la última semana. Estas cifras parecen indicar que uno de cada cinco personas que han consumido éxtasis en el último año, son consumidores habituales, es decir, semanales. Como en los casos anteriores, el consumo en universitarios es superior, tomando como referencia las últimos 30 días, que el de estudiantes de enseñanzas secundarias ( $3 \%$ y $1,1 \%$, respectivamente).

Los resultados sobre consumo de anfetaminas y alucinógenos muestran que son las sustancias recreativas menos consumidas, lo que puede estar indicando que el patrón de consumo más generalizado es experimental o esporádico. En el caso de la heroína, la prevalencia anual es del $0.8 \%$ y la semanal del $0,2 \%$, por lo que existe el patrón experimental en heroína, aunque en tasas muy bajas en esta población. En este caso, el consumo es levemente superior en población estudiante entre 14 y 18 años (ESTUDES, 2008).

En resumen, los resultados apuntan hacia que los jóvenes universitarios consumen más sustancias adictivas que los jóvenes en general y que los estudiantes de secundaria y bachillerato, que las sustancias más consumidas son el alcohol y el cannabis con un consumo principalmente semanal y que la cocaína y el éxtasis se consumen regularmente (semanalmente) por entre un $1,2 \%$ y un $1,8 \%$. Por otro lado, en los diferentes informes y estudios se 
habla del fenómeno del policonsumo, en el que parece claro que tener contacto con unas sustancias facilita tener contacto con otras y que contar con una actitud abierta a las drogas es un elemento que aumenta la probabilidad de consumir diferentes sustancias (Colder, Campbell, Ruel, Richarson y Flay, 2002; Calafat y cols., 2005; Espada, Hernández, Orgilés y Méndez, 2010).

Todo lo dicho señala que, especialmente los fines de semana, un porcentaje muy mayoritario de jóvenes universitarios consumen alcohol -sobre todo- y otras drogas. Este consumo puede haberse iniciado en periodos previos al universitario y consolidarse durante el mismo, o puede haberse iniciado durante el período universitario. En este contexto, y según los resultados obtenidos en las escalas validadas de consumo de alcohol y otras drogas utilizadas en este estudio, aproximadamente el $25 \%$ de la muestra presenta un perfil de riesgo de consumo de alcohol, y estos datos cuentan con garantías en cuanto a validez convergente con la Escala de Evaluación de Consumo de Alcohol y Otras Drogas utilizada en este estudio.

Estos resultados apuntan a que los problemas potenciales por abuso de alcohol son, con mucha probabilidad, los que aparecerán con más intensidad en el futuro de los jóvenes universitario actuales, en tanto que su consumo es más frecuente y en dosis más altas que ninguna de las sustancias ilegales evaluadas, y su toxicidad a corto, medio y largo plazo, es muy elevada. Este aspecto debe estar presente en relación con los objetivos y tipos de actuaciones relativos a la disminución del consumo y el riesgo derivado del mismo que lleven a cabo en el contexto universitario.

No obstante, a la hora de comparar estos resultados con los datos procedentes de otras fuentes hay que tener en cuenta que las diferencias metodológicas de los estudios y los distintos periodos en que se recaba la información, no nos permite más que apuntar posibles tendencias en las diferencias halladas. Por otro lado, las condiciones en que se ha llevado a cabo la recogida de datos: anonimato, remunerado y con recogida de orina para contrastar parte de la información recabada en el Autoinforme, puede haber influido en que los sujetos sean más sinceros y precisos en la información que proporcionan, y por tanto, haber aumentado la validez del autoinforme y los índices de prevalencia obtenidos.

Otro resultado de interés es que no se aprecian diferencias entre sexos en las distintas prevalencias tanto en el consumo de alcohol como de otras sustancias, es decir, aunque en los 
datos procedentes de otras fuentes no se informan resultados estadísticos de comparación entre sexos, en los porcentajes se observan mayores diferencias entre sexos que las que se han obtenido en este estudio. Este hecho señalaría que los patrones diferenciales de consumo entre sexos disminuyen en población joven y universitaria. Sin embargo, los hombres alcanzan en mayor proporción estados de embriaguez con alcohol y con alcohol y otras drogas que las mujeres. Es decir, los hombres y las mujeres beben alcohol con una frecuencia similar, pero ellos beben más cantidad de alcohol en cada ocasión.

A modo de conclusión, los resultados expuestos en este estudio y su relación con los obtenidos a través de las diferentes encuestas llevada a cabo por el Plan Nacional Sobre Drogas, así como los datos referidos a otros países europeos, permiten destacar que durante el periodo universitario se inicia y/o perpetúa el consumo de alcohol y otras drogas, lo que señala al contexto universitario como clave a la hora de diseñar e implementar actuaciones preventivas que modifiquen la actual incidencia y prevalencia del consumo de drogas entre estudiantes universitarios.

\section{Agradecimientos}

Esta investigación ha sido realizada gracias a la financiación de la Consejería de Igualdad y Bienestar Social de la Junta de Andalucía (España). 


\section{Referencias}

Babor, T., Higgins-Biddle, Saunders, J., \& Monteiro, M. (2001). AUDIT: Cuestionario de los trastornos debidos al consumo de alcohol. Pautas para su identificación en Atención Primaria. Ginebra: OMS.

Boland, M., Fitzpatrick, P. Scallan, E.,Daly, L. Herity, B. Horgan, J. y Bourke, G. Trends in medical students of tobacco, alcohol and drugs in an Irish university, 1973-2002. Drug and Alcohol Dependence, 85, 123-128.

Calafat, A., Fernández, C., Juan, M. y Becoña, E. (2005). Gestión de la vida recreativa: ¿Un factor de riesgo determinante en el uso reciente de drogas? Adicciones, 17, 4, 337-347.

Caspari, D. (1999). Cannabis and schizophrenia: results of a follow-up study. European Archives of Psychiatry and Clinical Neuroscience, 249, 45-49.

Colder, C., Campbell, R., Ruel, E., Richardson, J.L. y Fly, B. (2002). A finite mixture model of growth trajectoriesv of adolescent alcohol use: Predictors and consecuences. Journal of Consulting and Clinical Psychology, 70 (4), 976-985.

Espada, J.P., Hernández, O., Orgilés, M. y Méndez, X. (2010). Comparación de distintas estrategias para la modificación de la actitud hacia el consumo de drogas en escolares. Electronic Journal of Research in Educational Psychology, 8 (3), 1033-1050. Obtenido el 8 de Febrero de 2011 en:

http: investigación-psicopedagógica.org/revista/articulos/español/Art_22_468.pdf

European Monitoring Centre for Drugs and Drug Addiction (2009). The 2007 ESPAD report. Substance use among students in 38 countries. EMCDDA.

Hathaway, S.R. y McKinley, J.C. (1942): The Minnesota Multiphasic Personality Inventory, Nueva York: Psychological Corporation.

Infante, C. Barrio, G. y Martin, E. (2003). Tendencias, características y problemas asociados al consumo de drogas recreativas en España. Adicciones, 15, 2, 77-96.

Iversen, L.L. (2000). The science of marijuana. New York. Oxford University Press.

Laporte, J. (1979). El consumo de drogas en el medio universitario. En Actas del IX Congreso Internacional sobre tratamiento y prevención en drogodependencias. Madrid, Servicio de Estudios Sociales. (pag. 31-47).

Mayfield, D. McLeod, M.D. y Hall, P. (1974).The Cage Questionnare: Validation of a new Alcoholism Screening Instrument. American Journal Psychiatry, 131, 10, 1121-1123. 
McAndrew, C. (1965). The differentiation of male alcoholic outpatients from nonalcoholic psychiatric patients by means of the MMPI. Quarterly Journal of Studies on Alcohol, 26, 43-51.

Montero, I. y León, O.G. (2005). Sistema de clasificación del método en los informes de investigación en Psicología. Revista Internacional de Psicología Clínica y de la Salud/International Journal of Clínical and Health Psychology, 5, 1, 115-127.

Observatorio Español sobre Drogas (2003). Informe $n^{o}$ 6. Delegación del Gobierno para el Plan Nacional sobre Drogas.

Observatorio Español sobre Drogas (2006). Encuesta Domiciliaria sobre Abuso de Drogas en España (EDADES). Plan Nacional sobre Drogas.

Observatorio Español sobre Drogas (2007/2008). Encuesta Domiciliaria sobre Alcohol y otras Drogas en España (EDADES). Ministerio de Sanidad y Consumo. Delegación del Gobierno para el Plan Nacional Sobre Drogas.

Observatorio Español sobre Drogas (2008). Encuesta Estatal Sobre el Uso de Drogas en Estudiantes de Secundaria (ESTUDES). Ministerio de Sanidad y Politica Social. Delegación del Gobierno para el Plan Nacional Sobre Drogas.

Queipo, D., F.J. Álvarez y Velasco, A. (1986). Alcohol consumption among universitary students in Spain. Drug and Alcohol Dependence, 18, 41-49.

Rodríguez, J., Agulló, E y Agulló, M. S. (2003). Jóvenes, fin de semana y uso recreativo de drogas: evolución y tendencias del ocio juvenil. Adicciones, 15 supl.2, 7-33.

Rodríguez-Martos, A. Navarro, R.M., Vecino, C. y Perez, R. (1986). Validación de los cuestionarios KFA (CBA) y CAGE para el diagnóstico del alcoholismo. Drogalcohol, XI, 4, 132-139.

Roncero, J., Ramos, J.A., Collazos, F y Casas, M. (2001). Complicaciones psicóticas del consumo de cocaína. Adicciones, 13, (supl. 2) 179-189.

Rubio G, Bermejo, J, Caballero MC, Santo Domingo J. (1998). Validación de la para la identificación de trastornos por uso de alcohol (AUDIT) en atención primaria . Revista Clínica Española. 11-14.

Sainz, P.A., García-Portilla, P., Martínez, S., Bascarán, M.T., Bousoño, M y Bobes, J. (2003). Complicaciones psicopatológicas asociadas al consumo de drogas recreativas. Adicciones, 15, 2, 217-231.

Saunders, J.B., Aasland, O.G., Babor,T.F., De la Fuente, J.R. y Grant, M.T.I. (1993). Development of the alcohol use disorders identification test (AUDIT): WHO Collabora- 
tive project on Early Detection of persons with harmful alcohol consumption-II. Adicction, 88,791-804.

Viña, C. M. y Herrero, M. (2004). El consumo de sustancias psicoactivas en estudiantes de Psicología de la Universidad de la Laguna. Internacional Journal of Clinical and Health Psychology, 4, 521-536.

Webb, E., Ashton, C. H., Kelly, P. y Kamli, F. (1996). Alcohol and drugs use in UK university students. The lancet, 348, 5, 922-925.

Weed, N.C., Butcher, J.M., McKenna, T. Y y Ben-Porath, Y. (1992). New Measures for assessing Alcohol and Drug Abuse with the MMPI-2: The APS and AAS. Journal of Personality Assessment, 58, 2, 389-404. 